\title{
Different forms for nonlinear standing waves in deep water
}

\author{
by \\ Peter J. Bryant \\ Department of Mathematics, University of Canterbury, \\ Christchurch, New Zealand \\ and \\ Michael Stiassnie \\ Department of Civil Engineering, \\ Technion-Israel Institute of Technology, Haifa, Israel
}

No. 98

August, 1993 


\title{
Different forms for nonlinear standing waves in deep water
}

\author{
By Peter J. Bryant \\ Department of Mathematics, University of Canterbury, Christchurch, New Zealand \\ and Michael Stiassnie
}

Department of Civil Engineering, Technion-Israel Institute of Technology, Haifa, Israel.

Multiple forms for standing waves in deep water periodic in both space and time are obtained analytically as solutions of Zakharov's equation and its modification, and investigated computationally as irrotational two-dimensional solutions of the full nonlinear boundary value problem. The different forms are based on weak nonlinear interactions between the fundamental harmonic and the resonating harmonics of two, three, ... times the frequency and four, nine, ... respectively times the wavenumber. The new forms of standing waves have amplitudes with local maxima at the resonating harmonics, unlike the classical (Stokes) standing wave which is dominated by the fundamental harmonic. The stability of the new standing waves is investigated for small to moderate wave energies by numerical computation of their evolution, starting from the standing wave solution whose only initial disturbance is the numerical error. The instability of the Stokes standing wave to sideband disturbances is demonstrated first, by showing the evolution into cyclic recurrence that occurs when a set of nine equal Stokes standing waves is perturbed by a standing wave of a length equal to the total length of the nine waves. The cyclic recurrence is similar to that observed in the well known linear instability and sideband modulation of Stokes progressive waves, and is also similar to that resulting from the evolution of the new standing waves in which the first and ninth harmonics are dominant. The new standing waves are only marginally unstable at small to moderate wave energies, with harmonics which remain near their initial amplitudes and phases for typically 100-1000 wave periods before evolving into slowly modulated oscillations or diverging.

\section{Introduction}

Standing waves may be generated at the free surface of deep water contained between two parallel vertical walls. The most important feature is that their spectrum with respect to the coordinate perpendicular to the walls is discrete, with wave numbers which are integral multiples of $\pi / \ell$, where $\ell$ is the distance between the walls. If they are also periodic in time we describe them as pure standing waves, in contrast to standing waves which are evolving in time. 
Although Stokes investigated nonlinear progressive waves in the middle of last century, it appears that it was not until Rayleigh (1915) presented the third order theory that investigations of nonlinear standing waves at the free surface of deep water have been reported. Penney \& Price (1952) extended Rayleigh's method to the fifth order and applied it to a postulate that the stable standing wave of greatest height has a crest of right-angled nodal form. Taylor (1953) tested the results of Penney \& Price experimentally, showing reasonable agreement with their conclusions although he disagreed with parts of their arguments. Saffman \& Yuen (1979) used a numerical scheme which calculates the evolving position of the free surface to obtain wave profiles consistent with those predicted by Penney \& Price and observed by Taylor. It is noted that the standing waves observed by Taylor and calculated by Saffman \& Yuen are not pure standing waves. The different forms of standing waves described in the present investigation are pure standing waves, although in addition we do calculate time evolution properties of them.

Schwarz \& Whitney (1981) and Rottman(1982) developed high order expansions which were summed with the assistance of Padé approximants, with the aim in both investigations of understanding the approach to the wave of greatest height. The present investigation is restricted to standing waves of small to moderate heights. Mercer \& Roberts (1992) also studied steep standing waves using a distribution of vortices on the water surface, in a semi-Lagrangian approach. All of the above authors restricted themselves to pure standing waves with a Stokes ordering of harmonic amplitudes. Rottman (1982), however, indicated the possibility of different solutions with a different ordering of harmonic amplitudes, but did not pursue it in detail.

Glozman et al (1993) and Agnon et al (1992) derived and used a recursive high order Hamiltonian formulation of the water wave problem to study standing waves. Glozman $e t$ al focussed their computation on the interaction of the fundamental harmonic with the fourth harmonic in pure standing waves. They found, in addition to the Stokes type of wave, four new waves in which the fourth hamnonic is comparable to the fundamental harmonic. Agnon et al investigated all five waves (which they denoted S, A, B, C and D) using 12 to 20 harmonics, and found that all exist to high order. In the present investigation, we study the existence, stability, and long time behaviour of these and other new standing waves.

Okamura (1984) used Zakharov's equation, which is correct to the third order, to make calculations of the linear stability of pure standing waves with the Stokes ordering of harmonic amplitudes. The regions of instability of these waves to two- and three-dimensional standing wave disturbances were found. Although the mathematical description is consistent and the calculations at the lower wave steepnesses appear to be correct, there is a degree of abstraction in allowing the standing wave disturbances to have a continuous spectrum, unrelated to the discrete spectrum of the standing waves themselves. Also, the third order theory is not valid at the higher wave steepnesses considered. Stiassnie and Shemer (1984) modified Zakharov's 
equation in extending it to the fourth order. We use their improved formulation to find the regions of linear instability applicable to the different standing waves considered below.

\section{Theory}

\subsection{Background}

The equations governing the irrotational motion of waves on the free surface of deep water are

$$
\left.\begin{array}{c}
\nabla^{2} \phi=0, \quad z \leq \eta(\mathbf{x}, t), \\
\eta_{t}+\nabla \phi . \nabla \eta-\phi_{z}=0 \\
\phi_{t}+\frac{1}{2}(\nabla \phi)^{2}+g z=0
\end{array}\right\}, z=\eta(\mathbf{x}, t),
$$

where $\phi(\mathbf{x}, z, t)$ is the velocity potential, $\eta(\mathbf{x}, t)$ is the displacement of the free surface and $g$ the gravitational acceleration. The horizontal coordinates are $\mathbf{x}=(x, y)$, the vertical coordinate $z$ is pointing upwards, and $t$ is time.

When initial conditions are given in terms of $\eta(\mathbf{x}, 0), \phi(\mathbf{x}, \eta(\mathbf{x}, 0), 0)$, the problem can be transformed into an evolution equation in the Fourier plane

$$
i \frac{\partial B}{\partial t}=I_{3}(\mathbf{k}, t)+I_{4}(\mathbf{k}, t)+I_{5}(\mathbf{k}, t)+\ldots
$$

The new dependent variable $B(\mathbf{k}, t)$ is a free component of the wave field (as distinct from the bound components defined below), and $I_{3}, I_{4}, I_{5}, \ldots$ are integral operators representing quartet, quintet, sextet, ..., nonlinear interactions respectively.

The leading term $I_{3}$ on the right side of (2.2) was first derived by Zakharov (1968), the higher order term $I_{4}$ by Stiassnie \& Shemer (1984), with

$$
\begin{gathered}
I_{3}=\iiint_{-\infty}^{\infty} T_{0,1,2,3}^{(2)} B_{1}^{*} B_{2} B_{3} \delta\left(\mathbf{k}+\mathbf{k}_{1}-\mathbf{k}_{2}-\mathbf{k}_{3}\right) e^{i\left(\omega+\omega_{1}-\omega_{2}-\omega_{3}\right) t} d \mathbf{k}_{1} d \mathbf{k}_{2} d \mathbf{k}_{3}, \\
I_{4}=\iiint \int_{-\infty}^{\infty}\left\{U_{0,1,2,3,4}^{(2)} B_{1}^{*} B_{2} B_{3} B_{4} \delta\left(\mathbf{k}+\mathbf{k}_{1}-\mathbf{k}_{2}-\mathbf{k}_{3}-\mathbf{k}_{4}\right) e^{i\left(\omega+\omega_{1}-\omega_{2}-\omega_{3}-\omega_{4}\right) t}\right. \\
\left.+U_{0,1,2,3,4}^{(3)} B_{1}^{*} B_{2}^{*} B_{3} B_{4} \delta\left(\mathbf{k}+\mathbf{k}_{1}+\mathbf{k}_{2}-\mathbf{k}_{3}-\mathbf{k}_{4}\right) e^{i\left(\omega+\omega_{1}+\omega_{2}-\omega_{31}-\omega_{4}\right) t}\right\} d \mathbf{k}_{1} d \mathbf{k}_{2} d \mathbf{k}_{3} d \mathbf{k}_{4},
\end{gathered}
$$

where a compact notation is used in which the arguments $\mathbf{k}_{\mathbf{j}}$ are replaced by the subscript $i$ and the subscript zero is assigned to $\mathbf{k}$. The frequency $\omega$ is related to $\mathbf{k}$ through the linear dispersion relation $\omega(\mathbf{k})=(g|\mathbf{k}|)^{1 / 2}$. The kernels $T_{0,1,2,3}^{(2)}, U_{0,1,2,3,4}^{(2)}, \ldots$, as well as other kernels 
to appear subsequently, are given in Stiassnie \& Shemer (1984). The asterisk denotes the complex conjugate. The component $B(\mathbf{k}, t)$ is related to the Fourier transform (denoted by ${ }^{\wedge}$ ) of $\eta(\mathbf{x}, t)$ and of the velocity potential at the free surface, $\phi^{s}(\mathbf{x}, t)=\phi(\mathbf{x}, \eta(\mathbf{x}, t), t)$, through $b(\mathbf{k}, t)$ where

$$
\begin{gathered}
\hat{\eta}(\mathbf{k}, t)=\left(\frac{\omega}{2 g}\right)^{1 / 2}\left[b(\mathbf{k}, t)+b^{*}(-\mathbf{k}, t)\right], \\
\hat{\phi}^{s}(\mathbf{k}, t)=-i\left(\frac{g}{2 \omega}\right)^{1 / 2}\left[b(\mathbf{k}, t)-b^{*}(-\mathbf{k}, t)\right],
\end{gathered}
$$

and

$$
b(\mathbf{k}, t)=\left[B+B^{\prime}+B^{\prime \prime}+B^{\prime \prime \prime}+\ldots\right] e^{-i \omega(\mathbf{k}) t} .
$$

The quantities $B^{\prime}, B^{\prime \prime}, \ldots$, are the bound components of the wave field, which are given in terms of $B$ by equations such as

$$
\begin{gathered}
B^{\prime}=-\iint_{-\infty}^{\infty}\left\{V_{0,1,2}^{(1)} B_{1} B_{2} \delta\left(\mathbf{k}-\mathbf{k}_{1}-\mathbf{k}_{2}\right) \frac{e^{i\left(\omega-\omega_{1}-\omega_{2}\right) t}}{\omega-\omega_{1}-\omega_{2}}+V_{0,1,2}^{(2)} B_{1}^{*} B_{2} \delta\left(\mathbf{k}+\mathbf{k}_{1}-\mathbf{k}_{2}\right) \frac{e^{i\left(\omega+\omega_{1}-\omega_{2}\right) t}}{\omega+\omega_{1}-\omega_{2}}\right. \\
\left.+V_{0,1,2}^{(3)} B_{1}^{*} B_{2}^{*} \delta\left(\mathbf{k}+\mathbf{k}_{1}+\mathbf{k}_{2}\right) \frac{e^{i\left(\omega+\omega_{1}+\omega_{2}\right) t}}{\omega+\omega_{1}+\omega_{2}}\right\} d \mathbf{k}_{1} d \mathbf{k}_{2} .
\end{gathered}
$$

We consider two dimensional standing waves (independent of $y$ ) in the present investigation, in a deep tank with walls at $x=0, \ell$. The end conditions are

$$
\phi_{x}(0)=\phi_{x}(\ell)=0
$$

and $B(\mathbf{k}, t)$ has the discrete structure

$$
B(\mathbf{k}, t)=\sum_{m=1}^{M} B_{m}(t)[\delta(\mathbf{k}-m k \mathbf{i})+\delta(\mathbf{k}+m k \mathbf{i})]
$$

where $k=\pi / \ell$ and $\mathbf{i}$ is the unit vector in the $x$-direction. The upper bound $M$ is set to ensure that the sum stays within the gravity wave regime.

\subsection{Linear and weakly nonlinear theory}

The general solution of the linearized two dimensional version of (2.1) or (2.2) with the end conditions (2.6) is

$$
\eta=\sum_{m=1}^{M} a_{m} \cos (m k x) \cos \left[(m g k)^{1 / 2} t+\tau_{m}\right]
$$




$$
\phi=\sum_{m=1}^{M}-\left(\frac{g}{m k}\right)^{1 / 2} a_{m} e^{m k z} \cos (m k x) \sin \left[(m g k)^{1 / 2} t+\tau_{m}\right] .
$$

Each of the terms in (2.8a) is a free component, with amplitude $a_{m}$ and phase $\tau_{m}$. The steepness $m k a_{m}$ of each free component is of order $\epsilon \ll 1$ to justify the use of weakly nonlinear theory.

The general series $(2.8 \mathrm{a}, \mathrm{b})$ consist of standing waves with a common wavelength $2 \ell$ and a range of periods. We focus here on standing waves for which the motion is strictly periodic in time with a period $T$ where $T=2(\pi \ell / g)^{1 / 2}$, which we call pure standing waves. They are obtained when $a_{m}=0$ for all $m$ except when $m=n^{2}, n=1,2, \ldots$, and are given by

$$
\begin{gathered}
\eta=\sum_{n=1}^{N} a_{n^{2}} \cos \left(n^{2} k x\right) \cos \left[n(g k)^{1 / 2} t+\tau_{n^{2}}\right] \\
\phi=\sum_{n=1}^{N}-\frac{1}{n}\left(\frac{g}{k}\right)^{1 / 2} a_{n^{2}} e^{n^{2} k z} \cos \left(n^{2} k x\right) \sin \left[n(g k)^{1 / 2} t+\tau_{n^{2}}\right] .
\end{gathered}
$$

A large variety of standing waves of this type is possible because of the large range of possible values for $a_{n^{2}}$ and $\tau_{n^{2}}$.

Weakly nonlinear interactions produce standing wave solutions containing the series $(2.8 \mathrm{a}, \mathrm{b})$ (or $(2.9 \mathrm{a}, \mathrm{b})$ ) except that $a_{m}$ and $\tau_{m}$ are functions of the slow times $t_{p}=\epsilon^{p} t, p=2,3, \ldots$. The weakly nonlinear solutions also include the bound components composed from double, triple, ... products of the free components. For weakly nonlinear pure standing waves to exist with a strict periodicity like their linear counterpart $(2.9 \mathrm{a}, \mathrm{b})$, it is necessary that $a_{n^{2}}$ is independent of time and that $\tau_{n^{2}}$ has a specific time dependence. This constraint imposes selection criteria for $a_{n^{2}}$ from the third order theory and for $\tau_{n^{2}}$ from the fifth order theory, which are derived in $\S \S 2.3$ and 2.4 respectively.

The above weakly nonlinear standing waves also have slowly varying forms in which $a_{n^{2}}$ and $\tau_{n^{2}}$ are functions of the slow times, $t_{p}=\epsilon^{p} t, p=2,3, \ldots$. Side-band instability which brings in the components $a_{m}$ for $m=n^{2} \pm 1, n=2,3, \ldots$ produces slowly varying standing waves of this type. This phenomenon is discussed in $\$ 2.5$.

\subsection{Third order (Zakharov's) theory}

We consider weakly nonlinear pure standing waves containing three free components for which

$$
B(\mathbf{k}, t)=\sum_{n=1}^{3} B_{n^{2}}\left[\delta\left(\mathbf{k}-n^{2} k \mathbf{i}\right)+\delta\left(\mathbf{k}+n^{2} k \mathbf{i}\right)\right]
$$

where

$$
B_{n^{2}}=A_{n^{2}} e^{-i n \delta 2 t+i \phi_{n^{2}}}
$$


and $A_{n^{2}}, \Omega, \phi_{n^{2}}$ are all real constants with $A_{n^{2}}>0$. The period of this standing wave is

$$
T=\frac{2 \pi}{(g k)^{1 / 2}+\Omega} .
$$

We set $\phi_{1}=0$, without loss of generality.

Substitution of (2.10) into (2.2) with $I_{p}=0, p>3$ (Zakharov's equation) yields

$$
\begin{aligned}
& \Omega A_{1}=\left[\left(T_{1111}+2 T_{1 \overline{11}}\right) A_{1}^{2}+2\left(T_{1414}+T_{141 \overline{4}}\right) A_{4}^{2}+2\left(T_{1919}+T_{1 \S 1 \S}\right) A_{9}^{2}\right] A_{1} \\
& +T_{1 \bar{g} \overline{4} \overline{4}} A_{4}^{2} A_{9} e^{i\left(2 \phi_{4}-\phi_{0}\right)}, \\
& 2 \Omega A_{4}=\left[2\left(T_{4141}+T_{4 \overline{1} 4 \overline{1}}\right) A_{1}^{2}+\left(T_{4444}+2 T_{4 \overline{4} 4 \overline{4}}\right) A_{4}^{2}+2\left(T_{4949}+T_{4 \overline{4} 4 \overline{9}}\right) A_{9}^{2}\right] A_{4} \\
& +2 T_{449 \overline{1}} A_{1} A_{4} A_{9} e^{-i\left(2 \phi_{4}-\phi_{0}\right)} \text {, } \\
& 3 \Omega A_{9}=\left[2\left(T_{9191}+T_{9 \overline{19} \overline{1}}\right) A_{1}^{2}+2\left(T_{9494}+T_{9 \overline{4} 9 \overline{4}}\right) A_{4}^{2}+\left(T_{9899}+2 T_{9 \overline{9} 99 \overline{9})}\right) A_{9}^{2}\right] A_{9} \\
& +2 T_{9 \overline{1} 44} A_{1} A_{4}^{2} e^{i\left(2 \phi_{4}-\phi_{0}\right)} \text {. }
\end{aligned}
$$

The subscript notation is best explained by the example

$$
T_{9 \overline{1} 44}=T(9 k \mathbf{i},-k \mathbf{i}, 4 k \mathbf{i}, 4 k \mathbf{i}) .
$$

Most of the coefficients in the above system (2.12) are either zero or cancel each other, leaving the substantial simplification

$$
\begin{gathered}
\Omega A_{1}=-\frac{k^{3}}{4 \pi^{2}} A_{1}^{3}, \\
2 \Omega A_{4}=-\frac{64 k^{3}}{4 \pi^{2}} A_{4}^{3}, \\
3 \Omega A_{9}=-\frac{729 k^{3}}{4 \pi^{2}} A_{9}^{3} .
\end{gathered}
$$

The standing Stokes wave is a solution of (2.13) if $A_{4}=A_{9}=0$, when the well known result is obtained

$$
\Omega=-\frac{k^{3}}{4 \pi^{2}} A_{1}^{2}=-(g k)^{1 / 2} \frac{\left(k a_{1}\right)^{2}}{8} .
$$

This derivation uses the relation

$$
a_{m}^{2}=2\left(\frac{m k}{g}\right)^{1 / 2} \frac{A_{m}^{2}}{\pi^{2}}
$$

from Stiassnie \& Shemer (1984).

The system (2.13) admits four additional nontrivial solutions.

(i) For $A_{9}=0$, elimination of $\Omega$ between (2.13a) and (2.13b) gives

$$
\left(\frac{A_{4}}{A_{1}}\right)^{2}=\frac{1}{32}, \frac{a_{4}}{a_{1}}=\frac{1}{4}
$$

with $\Omega$ the same as in (2.14). 
(ii) For $A_{4}=0$, elimination of $\Omega$ between (2.13a) and (2.13c) gives

$$
\left(\frac{A_{9}}{A_{1}}\right)^{2}=\frac{1}{243}, \frac{a_{9}}{a_{1}}=\frac{1}{9}
$$

with $\Omega$ the same as in (2.14).

(iii) Equations (2.14), (2.15), and (2.16) remain valid for the more general case in which all three waves coexist. This result is easily generalized for $N>3$.

(iv) Another solution is possible for $A_{1}=0$. Elimination between (2.13b) and (2.13c) for this case yields

$$
\begin{gathered}
\Omega=-\frac{8 k^{3}}{\pi^{2}} A_{4}^{2}=-2(g k)^{1 / 2}\left(k a_{4}\right)^{2}, \\
\left(\frac{A_{9}}{A_{4}}\right)^{2}=\frac{32}{243}, \frac{a_{9}}{a_{4}}=\frac{4}{9} .
\end{gathered}
$$

Zakharov's equation, containing the lowest significant order of nonlinearity, enables specific values to be obtained for the wave amplitudes $a_{n^{2}}$. It does not provide any information about the phases $\phi_{n^{2}}$. For this, it is necessary to go to higher nonlinearities using the modified Zakharov equation and its extensions.

\subsection{Higher order nonlinearity}

Substitution of (2.10) into (2.2) with $I_{p}=0, p>4$ (modified Zakharov's equation) yields

$$
\begin{gathered}
\Omega A_{1}=-\frac{k^{3}}{4 \pi^{2}} A_{1}^{3}+\left[U_{1 \overline{1} \overline{1} \overline{1} 1}^{(2)} e^{-i \phi_{4}}+\left(U_{111 \overline{1} 4}^{(3)}+U_{1114 \overline{1}}^{(3)}\right) e^{i \phi_{4}}\right] A_{1}^{3} A_{4}, \\
2 \Omega A_{4}=-\frac{64 k^{3}}{4 \pi^{2}} A_{4}^{3}+U_{4 \overline{1} 111}^{(2)} e^{-i \phi_{4}} A_{1}^{4}, \\
3 \Omega A_{9}=-\frac{729 k^{3}}{4 \pi^{2}} A_{9}^{3} .
\end{gathered}
$$

Note that at this order of nonlinearity and for the three chosen components $A_{1}, A_{4}, A_{9}$, there is no additional coupling between the component $A_{9}$ and the other two. The new coupling between $A_{1}$ and $A_{4}$ could in principle produce information about the phase $\phi_{4}$ and produce a correction to (2.15). However, the numerical values for $U_{1 \overline{4} 1 \overline{1} \overline{1}}^{(2)}, U_{4 \overline{1} 111}^{(2)}, U_{111 \overline{1} 4}^{(3)}, U_{1114 \overline{1}}^{(3)}$ are extremely small (within the round-off error of our computation), so that the contribution of the new terms to the right side of (2.18) is negligible, if not zero.

The sextet nonlinear interaction term, $I_{5}$, in (2.2) has a similar structure to that of $I_{3}$ and $I_{4}$ in $(2.3 \mathrm{a}, \mathrm{b})$, with unknown real kernels which we denote by $Q_{0,1,2,3,4,5}$. This order modifies the structure of the system (2.18). An important part of the modification arises from the term

$$
Q_{4 \overline{4} 1 \overline{1} 1 \overline{1}} e^{-2 i \phi_{4}} A_{1}^{4} A_{4}
$$


on the right side of (2.18b). A necessary condition for a pure standing wave is therefore

$$
2 \phi_{4}=m \pi, m=0, \pm 1, \pm 2, \ldots
$$

which yields four different solutions having $\phi_{4}=0, \frac{\pi}{2}, \pi, \frac{3 \pi}{2}$, which we denote by standing waves SA, SB, SC, SD respectively. (The letter $\mathrm{S}$ indicates that the amplitude $a_{1}$ has a value comparable with that for the Stokes standing waves, and the second letter follows the notation of Agnon et al for the value of $\phi_{4}$.)

These different types of standing waves were first described by Agnon et al (1992). For $A_{4}=0, \quad A_{1}, A_{9} \neq 0$ only the two types of standing waves for which $\phi_{9}=0, \pi$ are found to occur, and are denoted by standing waves SNA, SNC respectively. (The letter $\mathbf{N}$ indicates that the amplitude $a_{4}$ is almost null.) We use numerical solutions of the full nonlinear wave problem in order to calculate these and other new forms of nonlinear standing waves and to investigate their stability.

\subsection{Linear stability}

The linear stability of the new waves to small disturbances at adjoining wavenumbers is studied next. Two cases are considered.

(i) Class I instability of the wave having components $A_{1}$ and $A_{9}$ with disturbances at $k_{8}=8 \mathrm{k}$ and $k_{10}=10 k$. This instability has the time scale $t_{2}=\epsilon^{2} t$. The governing equations are

$$
\begin{aligned}
& i \frac{d B_{8}}{d t}=T_{81098} B_{10}^{*} B_{9}^{2} e^{-i\left(2 \omega_{0}-\omega_{k}-\omega_{10}\right) t}, \\
& i \frac{d B_{10}}{d t}=T_{10}{ }_{899} B_{8}^{*} B_{9}^{2} e^{-i\left(2 \omega_{0}-\omega_{8}-\omega_{110}\right) t},
\end{aligned}
$$

where $B_{9}$ is given by $(2.10 \mathrm{~b})$ with $(2.14 \mathrm{a}, \mathrm{b})$ and $(2.16)$. Following Stiassnie \& Shemer (1984) or Okamura (1984), the range of steepness for instability, which is found to be independent of $\phi_{9}$ and thus the same for the standing waves SNA and SNC, is given by

$$
0.0812<k a_{1}<0.3239
$$

(ii) Class I combined with Class II instability of the wave having components $A_{1}$ and $A_{4}$ with disturbances at $k_{3}=3 k$ and $k_{5}=5 k$. This instability has the time scale $t_{3}=\epsilon^{3} t$ approximately. The governing equations are

$i \frac{d B_{3}}{d t}=T_{3544} B_{5}^{*} B_{4}^{2} e^{-i\left(2 \omega_{4}-\omega_{3}-\omega_{\mathrm{i}}\right) t}+\left(U_{35 \overline{4} 11}^{(2)}+U_{3 \overline{15} 141}^{(2)}+U_{3 \overline{15} 11 \overline{4}}^{(2)}\right) B_{5}^{*} B_{4} B_{1}^{2} e^{-i\left(2 \omega_{1}+\omega_{4}-\omega_{3}-\omega_{\mathrm{j}}\right) t}$, 
$i \frac{d B_{5}}{d t}=T_{5344} B_{3}^{*} B_{4}^{2} e^{-i\left(2 \omega_{4}-\omega_{i 3}-\omega_{i}\right) t}+\left(U_{5 \overline{3} 4 \overline{1} \overline{1}}^{(2)}+U_{5 \overline{1} \overline{1} 4 \overline{1}}^{(2)}+U_{5 \overline{3} \overline{1} \overline{1} 4}^{(2)}\right) B_{3}^{*} B_{4} B_{1}^{2} e^{-i\left(2 \omega_{1}+\omega_{4}-\omega_{3}-\omega_{i}\right) t}$

where $B_{1}$ and $B_{4}$ are given by $(2.10 \mathrm{~b})$ and $(2.14 \mathrm{a}, \mathrm{b}),(2.15)$. The range of steepness for instability depends on $\phi_{4}$. The standing wave SA is found to be unstable for

$$
0.2031<k a_{1}<0.3354
$$

the standing wave SC for

$$
0.1844<k a_{1}
$$

and the standing waves SB and SD are always unstable.

Both systems (2.21) and (2.22) represent cases of near-resonant interaction.

\section{Computation}

\subsection{Governing equations}

The dimensional quantities of the previous section are all made nondimensional for the purpose of computation. Lengths are made nondimensional with respect to $\ell / \pi$, where $\ell$ is the distance between the vertical walls, so that the standing wave motion takes place between the vertical planes $x=0$ and $x=\pi$. Times are made non-dimensional with respect to the inverse of the lowest linear frequency $(\pi g / \ell)^{1 / 2}$. The displacement of the water surface is written $z=\eta(x, t)$ and the water motion is assumed to be irrotational with the non-dimensional velocity potential $\phi(x, z, t)$. This satisfies Laplace's equation

$$
\phi_{x x}+\phi_{z z}=0, z \leq \eta
$$

with $\phi_{x}=0$ on $x=0$ and $x=\pi$, and $\phi_{z} \rightarrow 0$ as $z \rightarrow-\infty$. The nonlinear boundary conditions on the water surface are the kinematic condition

$$
\eta_{t}-\phi_{z}+\eta_{x} \phi_{x}=0 \text { on } z=\eta
$$

and the dynamic condition

$$
\phi_{t}+\eta+\frac{1}{2}\left(\phi_{x}^{2}+\phi_{z}^{2}\right)=0 \text { on } z=\eta
$$

One of the simplest nonlinear solutions is that for two-dimensional pure standing waves. Their Fourier series expansions (before truncation) are

$$
\eta=\sum_{m=1}^{\infty} \sum_{n=m \bmod 2}^{\infty} \cos m x\left(a_{m n} \cos n \omega t+b_{m n} \sin n \omega t\right), m+n \text { even }
$$


and

$$
\phi=\sum_{m=1}^{\infty} \sum_{n=m \bmod 2}^{\infty} \cos m x e^{m z}\left(c_{m n} \cos n \omega t+d_{m n} \sin n \omega t\right), m+n \text { even }
$$

where the coefficients $a_{m n}, b_{m n}, c_{m n}, d_{m n}$ are constants, and $\omega(\sim 1)$ is the nonlinear frequency of the fundamental harmonic. The constraint that $m+n$ is even is a consequence of the invariance of $\eta$ and $\phi$ when $x$ and $\omega t$ are both changed by $\pi$.

A number of measures of the nondimensional standing wave amplitude have been used previously. Solutions of Zakharov's equation have often used the steepness of the fundamental harmonic, $a_{11}$, while Schwartz and Whitney (1981) use half of the nondimensional wave height at $t=0$, which in the present notation is

$$
\epsilon_{S W}=\sum_{m=1}^{\infty} \sum_{n=1}^{\infty} a_{m n}, m, n \text { odd. }
$$

In order to reflect the fact that the present standing waves can have harmonics with local amplitude maxima at other than the fundamental harmonic, with phases different from that of the fundamental harmonic, the nondimensional root mean energy is chosen as the measure of wave amplitude. It is

$$
\epsilon=\left(\frac{2}{\pi} \int_{0}^{\pi}\left(\phi(x, \eta, t) \frac{\partial \eta}{\partial t}+\eta^{2}\right) d x\right)^{1 / 2}
$$

(West (1981), pp32-4), which is independent of $t$ because of the conservation of energy. Note that $\epsilon \sim a_{11}$ in the linear limit of a standing wave at the fundamental harmonic (with phase zero) only.

\subsection{Pure standing wave solutions (fixed point method)}

When the Fourier series $(3.2 \mathrm{a}, \mathrm{b})$ are substituted into $(3.1 \mathrm{~b}, \mathrm{c})$, denoted by $F=0, G=0$ respectively, the resulting equations may be rewritten

$$
F=\sum_{M=1}^{\infty} \sum_{N=M \bmod 2}^{\infty} \cos M x\left(A_{M N} \cos N \omega t+B_{M N} \sin N \omega t\right)=0, \quad M+N \text { even, }
$$

and

$$
G=\sum_{M=1}^{\infty} \sum_{N=M \bmod 2}^{\infty} \cos M x\left(C_{M N} \cos N \omega t+D_{M N} \sin N \omega t\right)=0, M+N \text { even }
$$

where the coefficients $A_{M N}, B_{M N}, C_{M N}, D_{M N}$ are functions of the coefficients $a_{m n}, b_{m n}, c_{m n}$, $d_{m n}$ and the frequency $\omega$. The function dependence could be found, but this is unnecessary for the calculation of numerical solutions. The Fourier series $(3.2 a, b)$ are also substituted into the 
normalising equation (3.4) rewritten to the left side, which is then denoted by $H=0$, and is a function of $a_{m n}, b_{m n}, c_{m n}, d_{m n}$ and $\omega$ also. This description assumes the usual calculation in which the root mean energy $\epsilon$ is given and the quantities $a_{m n}, b_{m n}, c_{m n}, d_{m n}$ and $\omega$ have to be found for a particular family of standing wave solutions. It takes only a simple re-ordering of the method if, instead, one of the latter quantities is given and $\epsilon$ is included among the quantities that have to be found.

Particular numerical values for the coefficients $a_{m n}, b_{m n}, c_{m n}, d_{m n}$ and $\omega$ are inserted into truncated versions of the Fourier series $(3.2 a, b)$, the series are substituted into $(3.1 b, c),(3.4)$ over an array of points in $x$ and $t$, and numerical values of the coefficients $A_{M N}, B_{M N}, C_{M N}, D_{M N}$ are calculated from truncated versions of $(3.5 \mathrm{a}, \mathrm{b})$ by the fast Fourier transform method. The partial derivatives of $A_{M N}, B_{M N}, C_{M N}, D_{M N}$ with respect to $a_{m n}, b_{m n}, c_{m n}, d_{m n}$ and $\omega$ are calculated numerically by the same method, and the Jacobian is formed from these derivatives and from the partial derivatives of $H$. The numerical values of $a_{m n}, b_{m n}, c_{m n}, d_{m n}$ and $\omega$ are improved then by Newton's method until $A_{M N}, B_{M N}, C_{M N}, D_{M N}, H$ are as close to zero as is required (typically $10^{-5}$ ). At the same time, the number of terms included in the truncated series may be increased until the residuals in $(3.5 \mathrm{a}, \mathrm{b})$ from terms not included are also as close to zero as is required.

The calculations are started when $\epsilon$ is sufficiently small that the coefficients $a_{m n}, b_{m n}, c_{m n}, d_{m n}$ may be given by linear values with $\omega=1$ as an initial approximation. Each solution for the coefficients is then used as an initial approximation to the next solution as $\epsilon$ is increased. The method of solution described above has evolved from one developed originally for three-dimensional permanent waves on deep water (Bryant, 1985). The generalization of the method to calculations of the nonlinear time evolution is described next.

\subsection{Non-periodic standing waves (time evolution method)}

The displacement, $\eta(x, t)$, and velocity potential, $\phi(x, z, t)$, are expanded now in Fourier series in $x$, with Fourier coefficients dependent on $t$, to yield (before truncation)

$$
\eta=\sum_{m=1}^{\infty} a_{m}(t) \cos m x
$$

and

$$
\phi=\sum_{m=1}^{\infty} b_{m}(t) e^{m z} \cos m x .
$$

When the Fourier series $(3.6 a, b)$ are substituted into $(3.1 b, c)$, these equations may be rewritten, following $(3.5 \mathrm{a}, \mathrm{b})$, in the form

$$
F=\sum_{M=1}^{\infty} A_{M}(t) \cos M x=0,
$$


and

$$
G=\sum_{M=1}^{\infty} B_{M}(t) \cos M x=0
$$

where the coefficients $A_{M}(t), B_{M}(t)$ are functionals of $a_{M}(t), b_{M}(t)$. Each of the coefficients, $A_{M}(t), B_{M}(t)$, when equated to zero, is an implicitly-defined, first order, nonlinear, ordinary differential equation for the corresponding Fourier coefficient of $(3.6 \mathrm{a}, \mathrm{b})$ in terms of all Fourier coefficients of $(3.6 a, b)$. It is extracted numerically from $(3.7 a, b)$ by the fast Fourier transform method. The set of differential equations obtained by equating all coefficients $A_{M}(t), B_{M}(t)$ to zero is solved numerically using an integrator devised for initial value problems in stiff systems of implicit, ordinary differential equations (NAG subroutine D02NGF), with a local error tolerance of $10^{-11}$. The differential equations are implicit because the exponential multipliers in (3.6b) are evaluated on the surface given by (3.6a).

The root mean energy (3.4) is calculated regularly as a check on the computation. In a number of the examples, the nonlinear interactions between the harmonics cause a slow transfer of energy to the higher harmonics. This results in a buildup of energy in the truncation harmonics, causing the total energy to increase and the calculations to fail. It is only a partial remedy to include more harmonics because it may only postpone the failure, and it may introduce rounding errors from the exponential multipliers in (3.6b) at large wave numbers.

The Fourier amplitudes calculated in $(3.6 \mathrm{a}, \mathrm{b})$ evolve in fast time. These Fourier expansions are interpreted more easily if the fast time variation is removed by Fourier decomposition over each fundamental period $2 \pi / \omega$, to yield (before truncation)

$$
\eta=\sum_{m=1}^{\infty} \sum_{n=0}^{\infty} a_{m n}(t) \cos m x \cos \left(n \omega t+\alpha_{m n}(t)\right)
$$

and

$$
\phi=\sum_{m=1}^{\infty} \sum_{n=0}^{\infty} c_{m n}(t) \cos m x e^{m z} \cos \left(n \omega t+\gamma_{m n}(t)\right)
$$

where the amplitudes and phases are now functions of slow time. (It should be noted that the amplitudes $a_{m n}, c_{m n}$ defined in $(3.8 \mathrm{a}, \mathrm{b})$ differ from those defined in $(3.2 \mathrm{a}, \mathrm{b})$ unless the phases are all zero.)

The nonlinear time evolution calculations are used to investigate the stability of pure standing wave solutions because they do not make the constant amplitude and phase assumptions implicit in linear stability analyses. The time evolution calculations are based on a numerical method independent of that used for the fixed point calculations, so that consistency between the properties obtained from the two sets of calculations increases confidence in both. 


\section{Standing wave examples}

\subsection{Standing waves with Stokes ordering}

The pure standing waves calculated originally by Rayleigh (1915), and improved to high order by Schwarz \& Whitney (1981), have an ordering of harmonic amplitudes similar to that of the Stokes progressive waves, and are named below as Stokes standing waves (denoted by $S)$. The amplitudes decrease monotonically, in general, as their wave number $m$ and frequency $n \omega$ increase.

The fixed point computational method (\$3.2) converges rapidly to the Stokes standing wave solutions with high numerical precision (error $<10^{-10}$ ), up to $\epsilon=0.2$. Solutions at larger values of $\epsilon$ are not included here because of the convergence difficulties associated with the exponential multipliers in (3.2b) at high wave numbers. By choosing the time origin so that the phase of the fundamental harmonic $\alpha_{11}$ is zero, all the Fourier sine coefficients in $(3.2 \mathrm{a}, \mathrm{b})$ are also zero. The frequency $\omega$, expanded as a polynomial in the fundamental amplitude $a_{11}$ over the range $0<\epsilon<0.2$ (using the NAG subroutine E02ADF), is found to be

$$
\omega=1.000000-0.1250 a_{11}^{2}-0.04 a_{11}^{4}+\ldots
$$

correct to 6 decimal places. The leading terms are consistent with (2.11) and (2.14a), and the corresponding expansion for $1 / \omega^{2}$ in terms of $\epsilon_{S W}$ (defined in (3.3)) agrees with Schwarz \& Whitney, equation (3.2), to 6 decimal places. For the purpose of comparison with later expansions, the amplitude $a_{42}$ is given by

$$
a_{42}=0.19 a_{11}^{4}-0.1 a_{11}^{6}+\ldots
$$

correct to 6 decimal places.

Although the Stokes standing waves are generally regarded as being stable at small to moderate amplitudes, they are unstable to subharmonic disturbances such as those described in $(2.21 \mathrm{a}, \mathrm{b}, \mathrm{c})$. The instability range (2.21c) suggests that if a set of 9 Stokes standing waves is generated between the vertical walls, with $\epsilon=0.1$ for each wave, the set is unstable to sideband disturbances at wave numbers 8 and 10. Initial conditions were chosen to consist of these 9 standing waves perturbed by a disturbance of amplitude 0.01 at wave number 1 , and their time evolution was calculated to confirm the initial linear instability and to find the form of the evolving standing wave motion. 


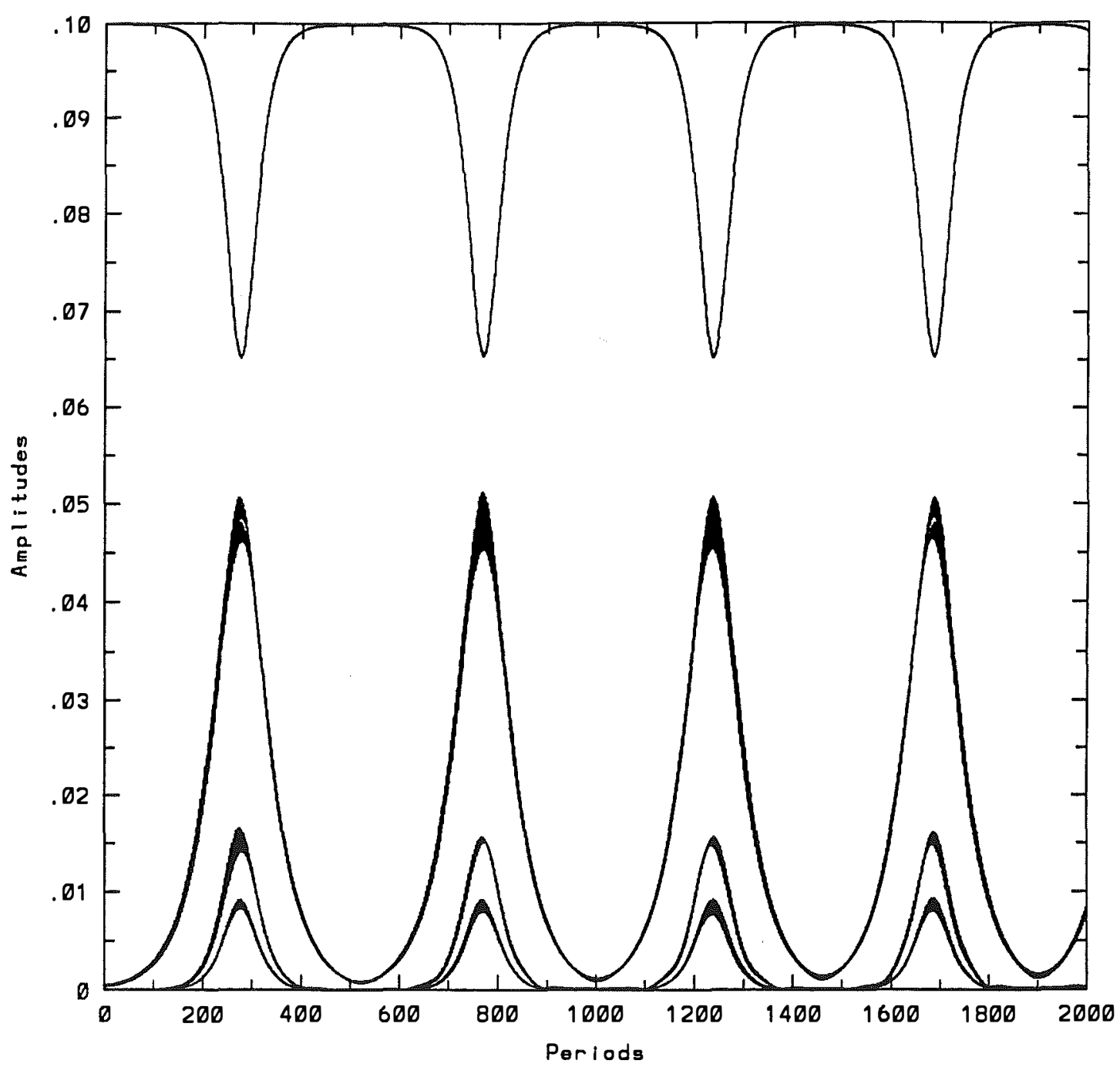

Figure 1. The sideband modulation of a set of 9 Stokes standing waves, where $\epsilon=0.1$ for each. The cyclic recurrence in slow time results from the instability of the Stokes standing waves to a subharmonic disturbance. The fundamental amplitude $a_{93}$ of each of the original standing waves is at the top, then in order the two sideband amplitudes $a_{83}$ and $a_{103}$ are almost superposed, followed in order by the sideband amplitudes $a_{113}$ and $a_{73}$. 
The expansions $(3.6 \mathrm{a}, \mathrm{b})$ are modified to

$$
\begin{gathered}
\eta=\sum_{m=1}^{45} a_{m}(t) \cos \frac{m x}{9} \\
\phi=\sum_{m=1}^{45} b_{m}(t) e^{m z / 9} \cos \frac{m x}{9}-
\end{gathered}
$$

because the standing wave motion takes place between $x=0$ and $x=9 \pi$. The fast time variation of the coefficients is removed by Fourier expansion over the corresponding period $6 \pi / \omega$, as in $(3.8 \mathrm{a}, \mathrm{b})$, to yield

$$
\eta=\sum_{m=1}^{45} \sum_{n=0}^{9} a_{m n}(t) \cos \frac{m x}{9} \cdot \cos \left(\frac{n \omega t}{3}-\alpha_{m n}(t)\right)
$$

and

$$
\phi=\sum_{m=1}^{45} \sum_{n=0}^{9} c_{m n}(t) \cos \frac{m x}{9} e^{m z / 9} \cos \left(\frac{n \omega t}{3}+\gamma_{m n}(t)\right),
$$

where the amplitudes and phases are now functions of slow time. (The chosen upper limits for the series are a balance between the needs for sufficient numerical precision and sufficient computational speed.)

The slow time evolution of the Stokes standing waves is illustrated in figure 1, which shows the fundamental amplitude $a_{93}$ of each of the original standing waves at the top, then in order the two sideband amplitudes $a_{83}$ and $a_{10} 3$ almost superposed, followed in order by the sideband amplitudes $a_{113}$ and $a_{73}$. The standing wave system is exhibiting cyclic recurrence, of similar form to that found for Stokes progressive waves (Lake et al (1977)). The system retains its initial Fourier amplitudes for about 100 wave periods while the linear instability gathers strength, then the amplitudes undergo the slow nonlinear modulation described usually as cyclic recurrence. Neither the period nor the amplitude of the nonlinear modulation cycles is constant. Each modulation cycle consists of about 150 wave periods in which the amplitudes remain close to their initial values and about 300 wave periods in which the wave amplitudes change more rapidly. The root mean energy (3.4) decreases by about $1 \%$ over the 2000 wave periods in the figure due to the energy losses at the truncation of the series (4.2).

In order to understand the nonlinear interactions better, the Fourier coefficients $a_{m}(t)$ in (4.2a), calculated in fast time over the complete modulation cycle of length 494 wave periods from period 274 to period 768, are decomposed into Fourier series of period equal to the 494 wave periods. (This Fourier decomposition, being over the large number $494 \times 64$ of points, is almost equivalent to regarding the frequencies as forming a continuum.) Most of the energy at wave number $m=9$ is found to be in a dominant waveband centred on frequency $n=3$, as is expected from the initial conditions. However, the energy at the sideband wave number $m=8$ is 
found to be mostly in a dominant waveband centred near the linear frequency $\omega(8)=\sqrt{8}$, rather than near the frequencies $n=2$ and $n=4$ expected from $(3.2 \mathrm{a}, \mathrm{b})$. The reason for the occurrence of the linear frequency is that the dominant sideband wave component is a free component, as defined in (2.2), not a bound component such as those in (3.2a,b). Bound components are found at this wave number, such as that at the frequency $2 \omega(9)-\omega(8)=6-\sqrt{8}$, but they have much less energy than the free component. Most of the energy at the other sideband wave numbers $m=7,10,11$ is also found in the free components with wavebands centred near $\omega(m)=\sqrt{m}, m=7,10,11$ respectively.

\subsection{Standing waves with resonating first and fourth harmonics}

The weakly nonlinear theory predicts that pure standing waves exist for which the first and fourth harmonics interact resonantly with the amplitude ratio (2.15)

$$
\frac{a_{42}}{a_{11}}=\frac{1}{4}
$$

in the present notation $(3.8 \mathrm{a}, \mathrm{b})$. The phase $\alpha_{42}$ relative to the phase $\alpha_{11}(2.20)$ may be $0, \pi / 2, \pi, 3 \pi / 2$, denoted by standing waves SA, SB, SC, SD respectively. The letter $S$ indicates that, like the Stokes standing wave, the amplitude $a_{11}$ is dominant, and the second letter denotes the phase $\alpha_{42}$ relative to $\alpha_{11}$. Standing waves of types SA and SC are described first.

The fixed point computational method (\$3.2) converges rapidly to these standing wave solutions with high numerical precision (error $<10^{-10}$ ), up to $\epsilon=0.2$. Like the Stokes standing waves, the time origin may be chosen so that all the Fourier sine coefficients in $(3.2 \mathrm{a}, \mathrm{b})$ are zero. In order to check the predictions of the weakly nonlinear theory, the frequency $\omega$ and resonant amplitude $a_{42}$ are expanded as polynomials in the fundamental amplitude $a_{11}$ over the range $0<\epsilon<0.05$. They are found to be

$$
\omega=1.000000-0.1250 a_{11}^{2}-0.08 a_{11}^{4}+\ldots
$$

correct to 6 decimal places, and

$$
a_{42}=0.25000 a_{11}-0.875 a_{11}^{3} \mp 0.01 a_{11}^{4}-0.9 a_{11}^{5}+\ldots,
$$

correct to 6 decimal places, where the upper sign refers to the SA wave and the lower sign to the SC wave. The phase $\alpha_{42}$ ( relative to $\alpha_{11}$ ) is zero for the SA waves and $\pi$ for the SC waves. The expansions confirm (2.14a) and (2.15), and (4.3b) has important differences from the corresponding expansion (4.1b) for Stokes standing waves. Equation (4.1b) begins with the fourth power, consistent with the Stokes ordering, while (4.3b) has linear and cubic leading terms due to the resonant interaction between the first and fourth harmonics. Apart from the 
maximum at $a_{42}$, the remaining harmonic amplitudes for the SA and SC waves lie close to a Stokes ordering.

The stability of the standing waves for root mean energies in the range $0<\epsilon<0.2$ is determined by calculating their long time evolution (\$3.3) for up to 10000 wave periods. At $\epsilon=0.05$, the harmonic amplitudes of both the SA and SC waves stay almost constant over the 10000 wave periods, but the phase $\alpha_{42}$ of the dominant fourth component relative to the phase $\alpha_{11}$ of the dominant first component drifts monotonically by more than $\pi$ over the same long time. This property is probably associated with the fact that the amplitudes are determined at the third order (2.15), but the phases are determined at the fifth order (2.19).

An unexpected long time behaviour occurs when the root mean energy is increased to $\epsilon=0.1$. The linear stability analysis for this case $(2.22 a, b, c)$ indicates that the standing waves SA and SC at this energy are stable. However, the time evolution of wave SA illustrated in figure 2, staring from the fixed point standing wave solution with the only disturbance being the initial numerical error, shows that wave SA is unstable. It shows also that the amplitudes of its dominant hannonics evolve into slow modulated nonlinear oscillations. Further, when the fast time variation of the sideband amplitudes in $(3.6 \mathrm{a}, \mathrm{b})$ is decomposed with respect to one modulation period equal to 3160 wave periods (effectively a Fourier decomposition into a continuum of frequencies), it is found that the energy in the sideband wave components occurs dominantly in wavebands centred near the bound components with amplitudes $a_{31}, a_{33}, a_{51}, a_{53}$, consistent with $(3.2 \mathrm{a}, \mathrm{b})$. Unlike the sideband modulation in $\$ 4.1$ or in the system (2.22), the energy in the free components of the sidebands does not rise above the background level here. This means that the sideband wave components play only a passive role in the nonlinear modulation illustrated in figure 2 , with the primary nonlinear interaction being that between the dominant resonating free components with amplitudes $a_{11}$ and $a_{42}$. We do not have a theoretical model yet to describe the instability leading to this nonlinear modulation.

Another property shown by figure 2 is that the standing wave SA is only marginally unstable. The time evolution illustrated in this figure is started from the fixed point standing wave solution, with the only disturbance being the initial numerical error. It can be seen that the standing wave amplitudes remain near their initial values for about 1000 wave periods before evolving into the nonlinear modulated oscillations. Although not shown in the figure, the phases also remain constant over the same 1000 wave periods. The root mean energy $\epsilon$ decreases by only $0.1 \%$ over the 10000 wave periods illustrated in figure 2. 

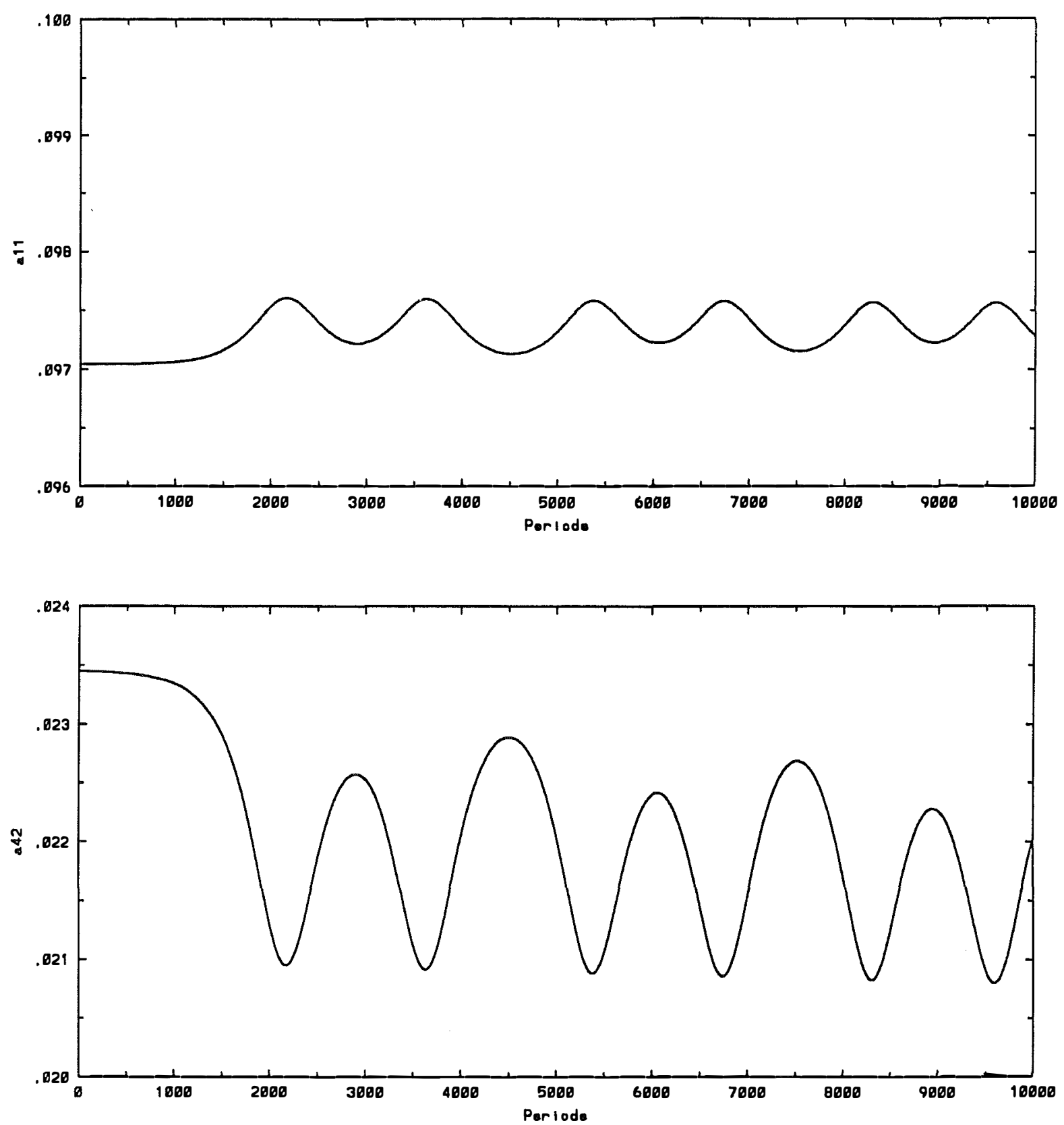

Figure 2. The time evolution of the resonant amplitudes $a_{11}$ and $a_{42}$, starting from the standing wave SA with $\epsilon=0.1$. 
When the root mean energy $\epsilon$ is increased further, the nonlinear modulation retains the same form as in figure 2 except that the modulation period decreases. For $\epsilon>0.16$ approximately, the nonlinear energy transfer to the higher harmonics causes the time evolution calculations to fail before the nonlinear modulation is established. The SC standing waves follow the same long time evolution except that the modulation periods are longer. The modulation period for the standing wave SC when $\epsilon=0.1$ is 4200 wave periods, compared with the 3160 wave periods of figure 2 .

It is not possible to calculate the harmonics of the SB and SD standing waves to the same high numerical precision, using the fixed point method (\$3.2), as it is for the SA and SC waves. When the numerical precision is set so that the error $<10^{-5}$, the fixed point method remains in the neighbourhood of a solution instead of converging to it. This appears to be a consequence of the weak dependence of the harmonics on their phases compared with the dependence on their amplitudes. The phases are fixed at zero or $\pi$ for the SA and SC waves, but a similar constraint is not applicable to the SB and SD waves. There is not sufficient numerical precision to obtain meaningful polynomial expansions for the frequency $\omega$ and the amplitude $a_{42}$ of the SB and SD waves in terms of the fundamental amplitude $a_{11}$. However, the precision is sufficient to observe that the frequency for the SB and SD waves is found to follow the same curve, within a difference less than $10^{-4}$, as that for the SA and SC waves over the range $0<\epsilon<0.2$. Also, the amplitude ratio $a_{42} / a_{11}$ for the SB and SD waves lies close to that for the SA and SC waves over the range $0<\epsilon<0.05(4.3 \mathrm{~b})$, but progressively departs from it at larger $\epsilon$.

When the long time evolution of the SB and SD waves is calculated at $\epsilon=0.05$, their harmonic amplitudes stays almost constant over the 10000 wave periods of the calculation, but like the SA and SC waves at the same value of $\epsilon$, the phase $\alpha_{42}$ of the dominant fourth component relative to the phase $\alpha_{11}$ of the dominant first component drifts from its initial value over the same long time. The SB and SD waves do not evolve into a nonlinear modulation of the harmonics at higher values of $\epsilon$ like the SA and SC waves, as illustrated in figure 2. Instead, for $\epsilon=0.1$, the harmonics remain constant in amplitude and phase for about 3000 wave periods, then the calculation progressively fails because the nonlinear transfer of energy to the higher harmonics causes energy accumulation at the truncation harmonics. The harmonics remain constant for about 200 wave periods at $\epsilon=0.15$, and only for about 30 wave periods at $\epsilon=0.2$, before failure of the calculations. The time intervals over which the wave amplitudes remain close to their initial values are of comparable length to those found for the SA and SC waves, indicating that all four types of standing waves are of comparable stability.

\subsection{Standing waves with resonating first and ninth harmonics}

The weakly nonlinear theory predicts that pure standing waves exist for which the first and 
ninth harmonics interact resonantly with the amplitude ratio (2.16)

$$
\frac{a_{93}}{a_{11}}=\frac{1}{9}
$$

in the present notation $(3.8 \mathrm{a}, \mathrm{b})$. The only pure standing waves of this type that have been predicted theoretically and found computationally have the values $0, \pi$ for the phase $\alpha_{93}$ relative to the phase $\alpha_{11}$. These two are denoted by standing waves SNA, SNC respectively. The letter $\mathrm{S}$ indicates that, as with the Stokes standing waves, the amplitude $a_{11}$ is dominant, the letter $\mathrm{N}$ indicates that the amplitude $a_{42}$ is almost null, and the third letter indicates that the phase $\alpha_{03}$ relative to $\alpha_{11}$ is zero or $\pi$ for the two solutions respectively.

The fixed point computational method (\$3.2) converges rapidly to the SNA and SNC standing wave solutions with high numerical precision (error $<10^{-10}$ ), up to $\epsilon=0.2$. Like the Stokes standing waves, the time origin may be chosen so that all the Fourier sine coefficients in $(3.2 a, b)$ for the SNA and the SNC waves are zero. The frequency $\omega$, expanded as a polynomial in the fundamental amplitude $a_{11}$ over the range $0<\epsilon<0.05$, is found to be

$$
\omega=1.000000-0.1250 a_{11}^{2}-0.04 a_{11}^{4}+\ldots,
$$

correct to 6 decimal places, for both the SNA and SNC standing waves. The amplitude $a_{93}$ is given by

$$
a_{93}=0.11111 a_{11}-1.230 a_{11}^{3} \pm 0.001 a_{11}^{4}+\ldots
$$

correct to 6 decimal places, where the upper sign refers to the SNA waves and the lower sign to SNC waves. The phase $\alpha_{93}$ ( relative to $\alpha_{11}$ ) is zero for the SNA waves and $\pi$ for the SNC waves. This expansion confirms (2.16) and has a similar form to (4.3b), with the linear and cubic leading terms arising from the resonant interaction between the first and ninth harmonics. Apart from the maximum near $a_{\bigcap 3}$, the remaining harmonic amplitudes for the SNA and SNC waves lie close to a Stokes ordering.

The stability of the SNA and SNC standing waves for root mean energies in the range $0<\epsilon<0.2$ is determined by calculating their long time evolution (\$3.3) for up to 10000 wave periods. At $\epsilon=0.05$, the harmonic amplitudes of both the SNA and SNC waves stay almost constant over the 10000 wave periods, but the phase $\alpha_{93}$ of the dominant ninth component relative to the phase $\alpha_{11}$ of the dominant first component drifts monotonically by more than $\pi$ over the same long time. Their long time behaviour at this value of $\epsilon$ is almost identical with that of the SA and SC waves. 


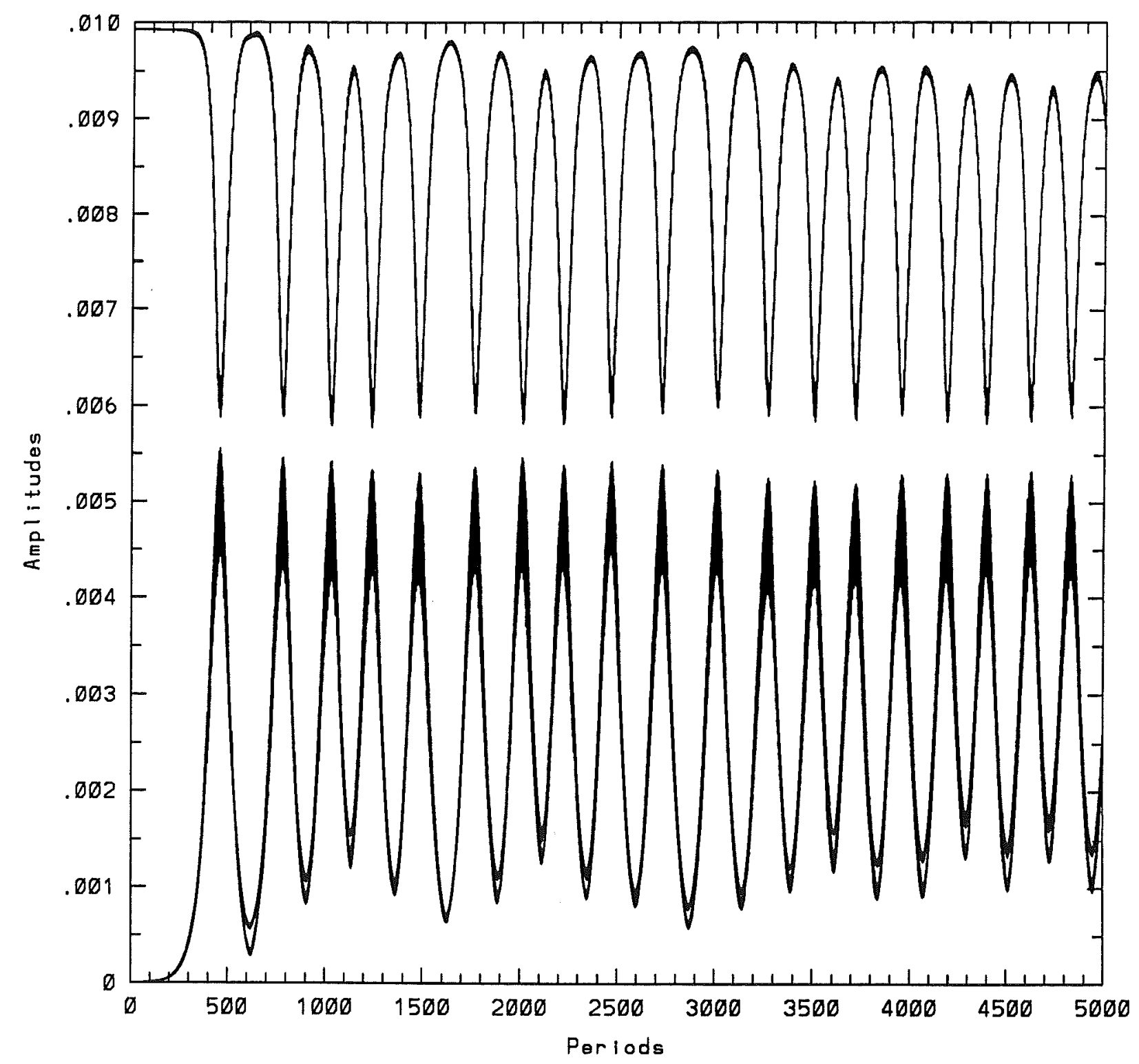

Figure 3. The time evolution of the resonant amplitude $a_{93}$ at the top, then in order the two sideband amplitudes $a_{83}$ and $a_{10} 3$ almost superposed, starting from the standing wave SNA with $\epsilon=0.1$. 
Unlike the SA and SC waves, a more predictable long time behaviour occurs when the root mean energy is increased to $\epsilon=0.1$. The linear stability analysis $(2.21 \mathrm{a}, \mathrm{b}, \mathrm{c})$ indicates that the standing waves SNA and SNC at this energy are unstable. The time evolution of the SNA wave at $\epsilon=0.1$ is illustrated in figure 3 starting from the fixed point standing wave solution, with the only disturbance being the initial numerical error. It can be seen that the standing wave amplitudes remain near their initial values for about 250 wave periods before evolving into nonlinear modulated oscillations. This initial behaviour is consistent with the instability predicted by (2.21c). When the fast time variation of the amplitudes in $(3.6 \mathrm{a}, \mathrm{b})$ is decomposed with respect to one modulation period equal to 301 wave periods (effectively a Fourier decomposition into a continuum of frequencies), it is found that the energy in the wave components during the modulated oscillations occurs dominantly in wavebands centred near the free components with frequencies $\omega(m)=\sqrt{m}, m=8,9,10$. The bound components of the sidebands which were dominant in the nonlinear modulated oscillations evolving from the SA and SC waves (\$4.2) have energies which are not much greater than the background level here. There are also bound components with small energies generated by the free components, such as those at wave number 9 with frequencies near $\sqrt{8} \pm 1, \sqrt{10} \pm 1$. The fundamental wave component with amplitude $a_{11}$ plays a passive role in the nonlinear modulated oscillations evolving from the SNA and SNC waves, and remains almost constant over the long time illustrated in figure 3. This property is in contrast to the nonlinear modulated oscillations evolving from the SA and SC waves, where it can be seen in figure 2 that the long time evolution of the amplitudes $a_{11}$ and $a_{42}$ is oscillatory and opposite in phase so that the total energy is conserved. The total energy in figure 3 decreases by less than $0.1 \%$ over the 10000 wave periods illustrated. It is noted that the nonlinear modulated oscillations evolving from the SNA and SNC waves have a similar structure to those evolving from the sideband instability of the set of 9 Stokes standing waves with the same value of $\epsilon(\$ 4.1$, figure 1$)$.

When the root mean energy $\epsilon$ is increased further, the nonlinear modulation retains the same form as in figure 3 except that the modulation period decreases. The SNC standing waves follow the same long time evolution as the SNA waves, with comparable modulation periods.

\subsection{Standing waves with resonating first, fourth and ninth harmonics}

It was shown in $\$ 2.3$ that the third order theory not only admits pure standing wave solutions in which resonant interactions occur between the two harmonics with amplitudes $a_{11}, a_{42}$ or with amplitudes $a_{11}, a_{93}$, but also those in which resonant interactions occur between all three harmonics with amplitudes $a_{11}, a_{42}, a_{93}$. The theory indicates that these standing waves have the same frequency relation (2.14a) and the same amplitude ratios $(2.15),(2.16)$ as the other two waves. 
A number of different standing wave solutions of this type have been found by the fixed point method (\$3.2) and their properties investigated by the time evolution method (\$3.3). If the known phase relations from the two previous sections are combined, there are in principle 8 different solutions of this type. The phase $\alpha_{42}$ relative to the phase $\alpha_{11}(2.20)$ may be $0, \pi / 2, \pi, 3 \pi / 2$, and the phase $\alpha_{93}$ relative to the phase $\alpha_{11}$ may be $0, \pi$. The solutions are denoted SAA, SAC, SCA, SCC, SBA, SBC, SDA, SDC, with an obvious notation that combines the previous notations. The first 4 solutions, in which all phase differences are $0, \pi$, have Fourier expansions $(3.2 \mathrm{a}, \mathrm{b})$ with no sine terms. The only solution of the last 4 which has been found with confidence is the wave SBC, and its properties are similar to those of the first 4 waves.

The properties of the wave SAC are described now, as a representative of the above 5 standing waves. The fixed point computational method (\$3.2) converges rapidly to the SAC standing wave solutions with high numerical precision (error $<10^{-10}$ ). The polynomial expansions for the frequency and the resonant amplitudes of the wave SAC over the range $0<\epsilon<0.05$ are

$$
\begin{gathered}
\omega=1.000000-0.1250 a_{11}^{2}-0.09 a_{11}^{4}+\ldots, \\
a_{42}=0.25000 a_{11}-0.786 a_{11}^{3}+0.07 a_{11}^{4}+\ldots \\
a_{93}=0.11111 a_{11}-1.107 a_{11}^{3}-0.07 a_{11}^{4}+\ldots,
\end{gathered}
$$

all correct to 6 decimal places. The phase $\alpha_{93}$ ( relative to $\alpha_{11}$ ) is $\pi$ for the SAC waves. The two leading terms of the expansion for $\omega$ agree with (2.14a), and the leading terms of the expansions for $a_{42}$ and $a_{93}$ agree with (2.15) and (2.16) respectively.

The stability of the SAC standing wave for root mean energies in the range $0<\epsilon<0.2$ is determined by calculating their long time evolution (\$3.3). At $\epsilon=0.05$, the harmonic amplitudes stay almost constant over the lu000 wave periods of the calculation, but the phases $\alpha_{42}, \alpha_{93}$ of the dominant components relative to the phase $\alpha_{11}$ of the first component drift over the same long time. The long time evolution when the root mean energy is increased to $\epsilon=0.1$ is illustrated in figure 4 , starting from the fixed point standing wave solution with the only disturbance being the initial numerical error. It can be seen that the standing wave amplitudes remain near their initial values for about 400 wave periods before evolving into nonlinear modulated oscillations. Their subsequent evolution consists of the slow change observed in figure 2 for the amplitudes $a_{11}$ and $a_{42}$, superposed on the the faster modulation observed in figure 3 as the amplitude $a_{93}$ interacts independently with the free components in its sidebands. The root mean energy $\epsilon$ changes by less than $0.1 \%$ over the first 3500 wave periods illustrated, and then by about $0.3 \%$ over the last 1500 wave periods associated with the change in appearance of the modulation. 

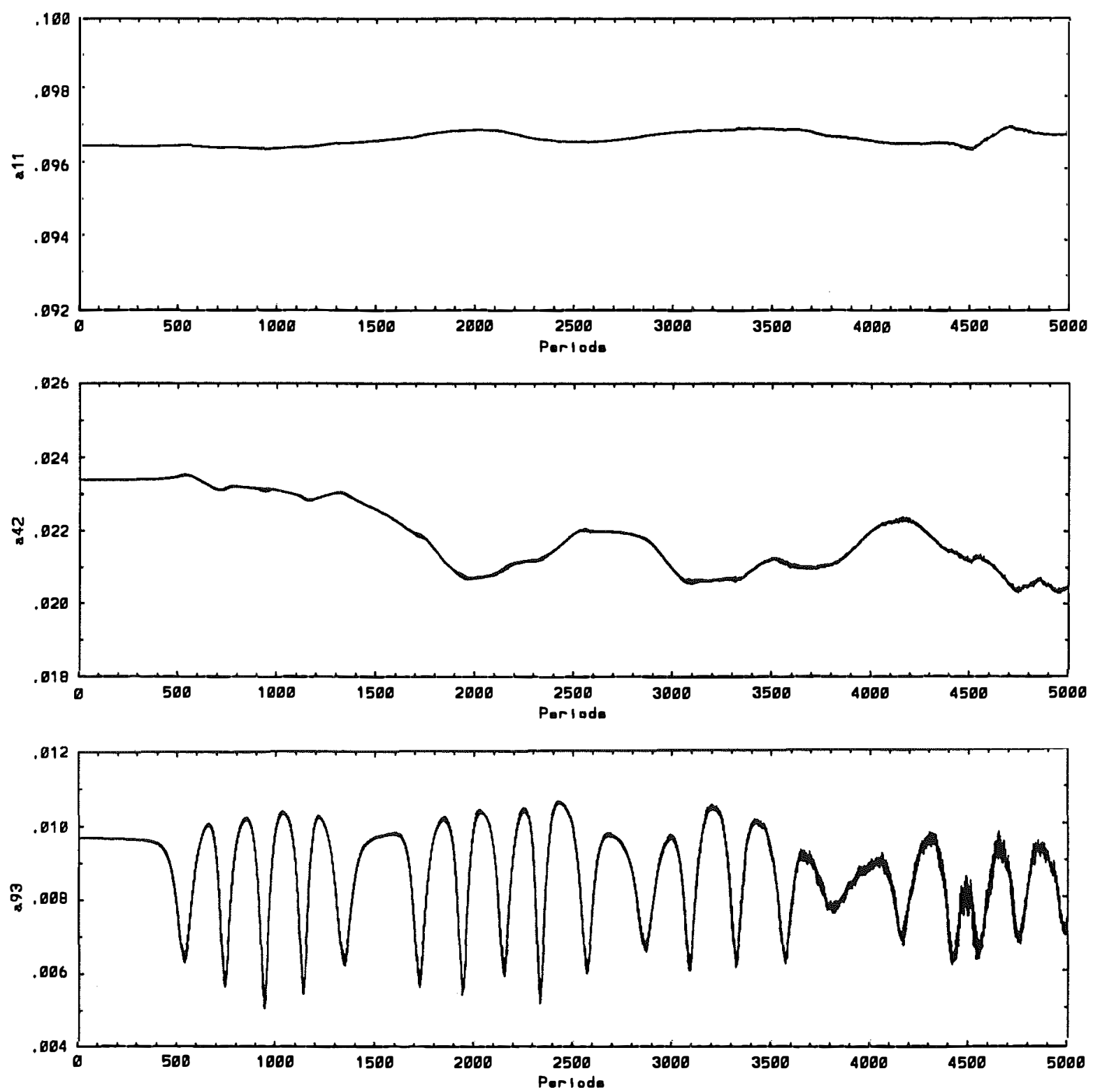

Figure 4. The time evolution of the resonant amplitudes $a_{11}, a_{42}$ and $a_{93}$, starting from the standing wave SAC with $\epsilon=0.1$. 
When the root mean energy is increased to $\epsilon=0.15$, the SAC standing wave amplitudes remains near their initial values for about 100 wave periods. They then begin nonlinear modulated oscillations similar to those in figure 4 except that the modulation periods are smaller. These terminate after a further 250 wave periods when the nonlinear energy transfer to the higher harmonics causes the time evolution calculations to fail.

\subsection{Standing waves with resonating fourth and ninth harmonics}

The third order theory in $\$ 2.3$ not only admits pure standing wave solutions in which resonant interactions occur between the two harmonics with amplitudes $a_{11}, a_{42}$ or with amplitudes $a_{11}, a_{93}$, but also those in which resonant interactions occur between the two harmonics with amplitudes $a_{42}, a_{93}$ while the fundamental amplitude $a_{11}$ remains much smaller. The theory (2.17b) indicates that these standing waves have an amplitude ratio

$$
\frac{a_{93}}{a_{42}}=\frac{4}{9}
$$

The standing wave solutions of this type have been found by the fixed point method (\$3.2) and their properties investigated by the time evolution method (\$3.3). The argument based on phases advanced in the previous section indicates that there are in principle 8 different solutions of this type. The only 2 solutions of this type that have been found with any confidence are denoted by NAC and NCC, where N indicates that the amplitude $a_{11}$ is almost null, the second letter indicates that the phase $\alpha_{42}$ relative to $\alpha_{11}$ is zero or $\pi$ in the two solutions, and the third letter indicates that the phase $\alpha_{93}$ relative to $\alpha_{11}$ is $\pi$ for both solutions. Although $a_{11} \ll a_{42}$ for small $\epsilon(<0.05), a_{11}$ increases as $\epsilon$ increases, becoming comparable with $a_{42}$ at about $\epsilon=0.1$.

The properties of the wave NAC are described now, as a representative of both standing waves of this type. The fixed point computational method (\$3.2) converges rapidly to the NAC standing wave solutions with high numerical precision (error $<10^{-10}$ ). The polynomial expansions with respect to $a_{42}$ for the frequency and for $a_{93}$ over the range $0<\epsilon<0.05$ are

$$
\begin{gathered}
\omega=1.00000-2.072 a_{42}^{2}+5.90 a_{42}^{3}+\ldots \\
a_{93}=0.4445 a_{42}-0.053 a_{42}^{2}+\ldots
\end{gathered}
$$

correct to 5 decimal places. The phase $\alpha_{93}$ ( relative to $\alpha_{11}$ ) is $\pi$ for the NAC waves. 


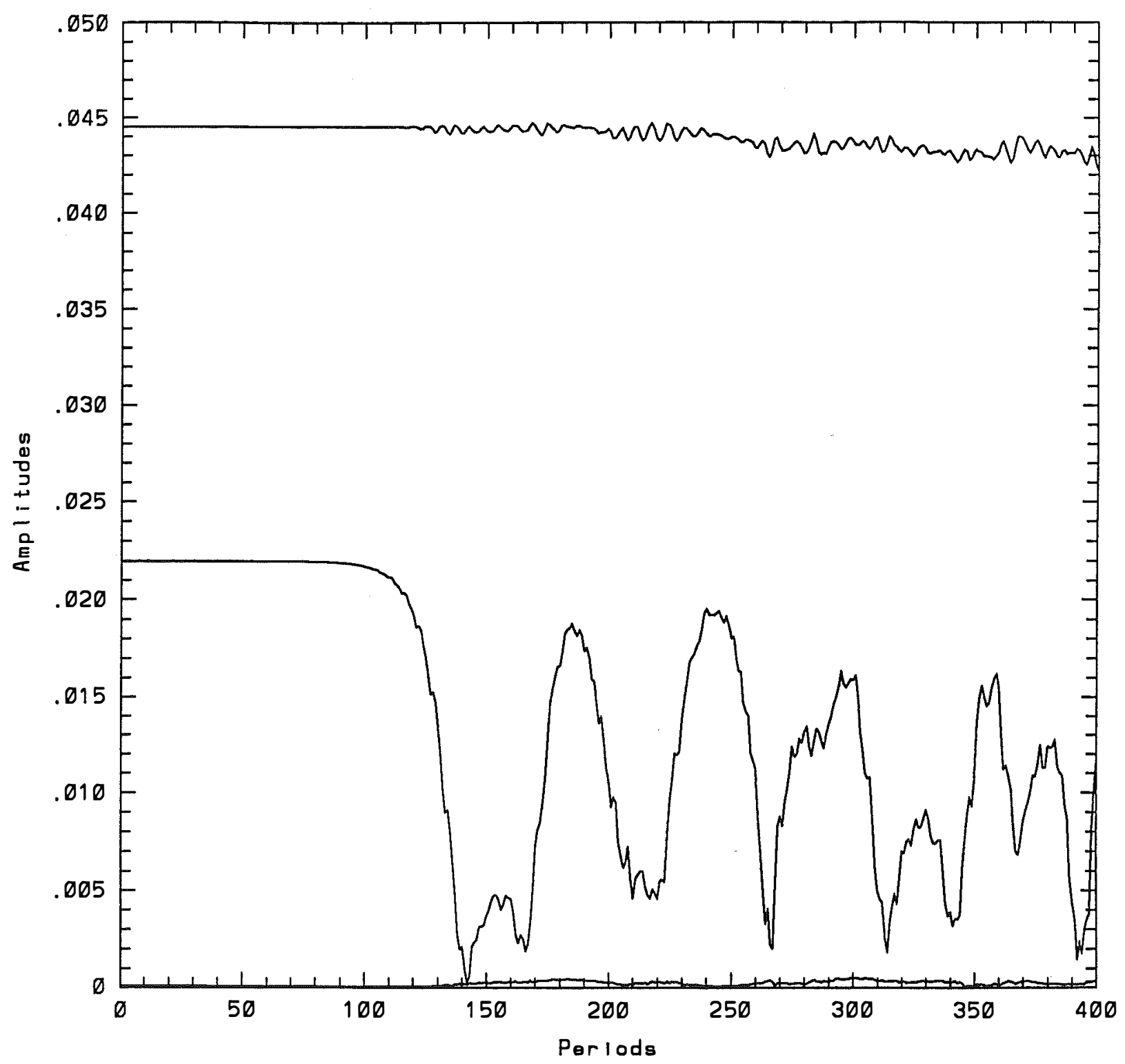

Figure 5. The time evolution of the amplitudes $a_{42}$ at the top of the figure, $a_{93}$ next, and $a_{11}$ (which is so close to zero that it is barely visible at the bottom of the figure), starting from the standing wave NAC with $\epsilon=0.05$. 
Better agreement with the weakly nonlinear theory is found with the corresponding relations at $\epsilon=0.001$, which are

$$
\begin{gathered}
\omega=1.00000-2.0000 a_{42}^{2} \\
a_{93}=-0.44446 a_{42} .
\end{gathered}
$$

Equation (4.6c) agrees with (2.17a) and (4.6d) agrees with (2.17b).

The stability of the NAC standing wave for root mean energies in the range $0<\epsilon<0.1$ is determined by calculating their long time evolution (\$3.3). Because the amplitude $a_{11}$ is much smaller than previously, it is necessary to choose the root mean energy $\epsilon$ to be smaller than in the previous examples so that the other resonant amplitudes have values comparable with those chosen previously. For this reason, the value chosen for the long time evolution illustrated in figure 5 is only $\epsilon=0.05$. It can be seen that the standing wave amplitudes remain near their initial values for about 100 wave periods before evolving into nonlinear modulated oscillations. Their subsequent evolution is similar to that illustrated in figure 3. The amplitudes $a_{11}$ and $a_{42}$ are almost passive, while the amplitude $a_{93}$ interacts independently with the free components in its sidebands. The root mean energy $\epsilon$ changes by about $0.8 \%$ over the 400 wave periods illustrated, but remains constant over the initial 100 wave periods while the standing wave stays near its initial amplitudes.

When the root mean energy is increased to $\epsilon=0.1$, the NAC standing wave amplitudes remain near their initial values for about 20 wave periods before the nonlinear energy transfer to the higher harmonics causes the time evolution calculations to fail.

\section{Summary and Discussion}

The Stokes standing wave $S$ was the only known pure standing wave until Agnon et al (1992) found four new waves, denoted by us SA, SB, SC, and SD. In the present paper we have studied these four new waves, and presented nine additional different forms of pure standing waves, namely SNA, SNC; SAA, SAC, SCA, SCC, SBC; NAC, NCC. This is not expected to be a complete list, and we discuss the rather peculiar combination SAAA . . below.

The agreement between our two approaches: (i) weakly nonlinear theory, and (ii) fully nonlinear computation, ensures that these solutions are genuine. We have shown that the solutions are not less stable than the original Stokes standing waves, and thus deserve attention in future investigations and applications. For example, the Fourier amplitudes of the standing wave SA in figure 2 remain close to their initial values for over 1000 wave periods before they evolve into a slow modulation. The sideband modulation is of special interest because it exhibits the 
same form of cyclic recurrence as the Stokes progressive waves. The question of the conditions for the generation of the new standing waves in a more realistic forced and damped system is an important objective for a future study.

The presence of significant higher harmonics in the new standing waves becomes of greater importance for the water surface slope and acceleration. This is demonstrated with an exaggerated extension (ignoring surface tension and viscosity) to a possible wave SAAA .... The free surface of this 'wave' is

$$
\eta=a_{1} \sum_{n=1}^{\infty} \frac{1}{n^{2}} \cos \left(n^{2} k x\right) \cos \left[n(g k)^{1 / 2} t\right],
$$

obtained as a generalization of combining (2.9a) with (2.15), (2.16). The energy of this 'wave' is approximately

$$
\frac{1}{4} \rho g a_{1}^{2} \sum_{n=1}^{\infty} \frac{1}{n^{4}}=\frac{1}{4} \rho g a_{1}^{2} \frac{\pi^{4}}{90}=1.082\left(\frac{1}{4} \rho g a_{1}^{2}\right),
$$

which is only about $8 \%$ greater than the energy of the fundamental hamonic given in parentheses. However, the vertical acceleration of the free surface $\left(\sim \partial^{2} \eta / \partial t^{2}\right)$ diverges almost everywhere. The geometry of $\eta$ at any given time is rather complicated. At $t=0$, it takes the form of Riemann's continuous but nondifferentiable function (Mandelbrot (1982))

$$
R=\sum_{n=1}^{\infty} \frac{1}{n^{2}} \cos \left(n^{2} \zeta\right)
$$

which can be shown to be a multifractal function with a dimension $\approx 1.15$.

M.S. acknowledges the support of the U.S. Office of Naval Research, Contract N00014-91-J-1449, and the award of an Erskine Visiting Fellowship at the University of Canterbury. He is grateful to the Head and staff of the Mathematics Department, University of Canterbury, for their hospitality. 


\section{REFERENCES}

Agnon, Y., Dold, J. W., Glozman, M., MLman, Tal \& StIASSNIE, M. 1992 New standing water-wave solutions. In Order and Chaos in Standing Waves, second scientific report, Coastal and Marine Engineering Research Institute, Technion, Haifa, Israel. P.N. 305/92.

BRYANT, P. J. 1985 Doubly periodic progressive permanent waves in deep water. J. Fluid Mech. 161, 27-42.

Glozman, M., Agnon, Y. \& StIASSNIE, M. 1993 High order formulation of the waterwave problem. To appear in Physica $D$.

LAKe, B. M., Yuen, H. C., RungaldieR, H. \& Ferguson, W. E. 1977 Nonlinear deepwater waves: theory and experiment. Part 2. Evolution of a continuous wave train. J. Fluid Mech. 83, 49-74.

MANDELBROT, B. 1982 The Fractal Geometry of Nature. Freeman, San Francisco.

MERCER, G. N. \& ROBERTS, A. J. 1992 Standing waves in deep water: Their stability and extreme form. Phys Fluids A 4, 259-269.

OKAMURA, M. 1984 Instabilities of weakly nonlinear standing gravity waves. J. Phys Soc. Japan 53, 3788-3796.

PENNEY, W. G. \& PRICE, A. T. 1952 Finite periodic stationary gravity waves in a perfect liquid. Phil. Trans R. Soc. Lond. A 244, 254-284.

RAYLEIGH, J. W. S. 1915 Deep water waves, progressive or stationary, to the third order of approximation. Proc. R. Soc. Lond. A 91, 345-353.

ROTTMAN, J. W. 1982 Steep standing waves at a fluid interface. J. Fluid Mech. 124, 283-306.

SAFFMAN, P. G. \& YUEN, H. C. 1979 A note on numerical computations of large amplitude standing waves. J. Fluid Mech. 95, 707-715.

SCHWARTZ, L. W. \& WHITNEY, A. K. 1981 A semi-analytic solution for nonlinear standing waves in deep water. J. Fluid Mech. 107, 147-171.

STIASSNIE, M. \& SHEMER, L 1984 On modifications of the Zakharov equation for surface gravity waves. J. Fluid Mech. 143, 47-67.

TAYLOR, G. I. 1953 An experimental study of standing waves. Proc. R. Soc. Lond. A 218, 44-59.

WeST, B. J. 1981 Deep water gravity waves. Lecture Notes in Physics, 146. Springer ZAKHAROV, V. E. 1968 Stability of periodic waves of finite amplitude on the surface of a deep fluid. J. Appl. Mech. Tech. Phys. 2, 190-194. 